\title{
Erweiterte Horizonte - Ein technischer Blick in die Zukunft der Arbeit
}

\author{
Benedikt Mättig, Jana Jost und Thomas Kirks
}

\subsection{Projektbeschreibung}

Der Mensch stellt auch in der Produktion und Logistik von morgen einen entscheidenden Faktor dar. Aus der immer weiter wachsenden Datenmenge in Unternehmensprozessen müssen jene Informationen extrahiert werden, welche den Mitarbeiter bei seiner Arbeit unterstützen können und nicht zu Überforderung führen.

Das durch das BMBF geförderte Forschungsprojekt SmARPro (Smart Assistance for Humans in Production Systems) befasst sich mit dieser Problematik. Es wird ein System entwickelt, das über einheitliche und standardisierte Schnittstellen Daten aller umgebenden Systeme erfasst und diese in der SmARPro-Plattform zu kontextsensitiven Informationen aufbereitet. Diese werden dem Mitarbeiter über Wearables wie beispielsweise Datenbrillen, Smart Watches, Smartphones oder Tablets angezeigt.

Den Fokus des Projektes bildet die Integration des Menschen in komplexe Systeme und Prozesse, ohne ihn dabei durch die Vielzahl an prozessspezifischen Informationen zu überfordern. Um dieses Ziel zu erreichen, werden in SmARPro individuell an die jeweilige Person angepasste Informationsdarstellungen verwendet. Diese basieren auf Informationen zum Kontext des Mitarbeiters sowie auf individuellen Eigenschaften und Bedürfnissen der jeweiligen Person. Der Kontext setzt sich aus Parametern wie Ort, Zeit und Rolle des Mitarbeiters zusammen. Beispielsweise würde bei einem Maschinenbediener in der Produktion exakt ermittelt werden, wo er sich an der Maschine befindet und welche

B. Mättig $(\triangle) \cdot$ J. Jost · T. Kirks

Fraunhofer-Institut für Materialfluss und Logistik IML, Joseph-von-Fraunhofer-Str. 2-4, 44227

Dortmund, Deutschland

e-mail: benedikt.maettig@iml.fraunhofer.de; jana.jost@iml.fraunhofer.de; thomas.kirks@iml.fraunhofer.de 
Informationen er an seinem derzeitigen Arbeitsplatz zum aktuellen Zeitpunkt benötigt. Hinzu kommen individuelle Parameter, die neben der bevorzugten Sprache und altersabhängigen Darstellungseigenschaften auch persönlich bevorzugte Darstellungsformen des Mitarbeiters berücksichtigen. Aus all diesen Parametern werden in SmARPro Darstellungsmodelle definiert, die für jede Person und jeden Kontext vorgeben, welche Informationen auf welche Weise dargestellt werden sollen.

Neben der Art der Darstellung ist die Wahl der darzustellenden Informationen für einen funktionierenden Prozess entscheidend. Innerhalb des Projektes werden eingehende Daten durch die Plattform, basierend auf kontextabhängigen Parametern, so aufbereitet, dass nur die Informationen an den Mitarbeiter weitergegeben werden, die er zum aktuellen Zeitpunkt benötigt. Hierbei muss berïcksichtigt werden, dass fehlende Informationen zu fehlerhaften Prozessen führen können. Darüber hinaus verursacht eine zu große Menge an Informationen, dass der Mitarbeiter länger für deren Aufnahme und Interpretation benötigt. Zudem kann es durch die Informationsflut dazu kommen, dass wichtige Informationen nicht die benötigte Aufmerksamkeit des Mitarbeiters erhalten.

Durch die individuell angepasste Informationsdarstellung sowie die Reduktion der dargestellten Informationen auf die wesentlichen Elemente, kann die Informationsaufnahme schneller erfolgen. Dies ermöglicht eine schnellere Prozessbearbeitung durch weniger Unterbrechungen und erhöht außerdem die Akzeptanz des Benutzers, durch eine Reduktion des Frustfaktors für den Mitarbeiter.

\subsection{Anwendungsfall}

Die in diesem Projekt betrachteten Berufe und Tätigkeiten werden in feingranulare, prozessbezogene Arbeitsschritte aufgeteilt und analysiert. Ziel dieser Analyse ist die Identifizierung von Einsatzfällen für Wearables. Die verwendeten Technologien bieten die unterschiedlichsten Vorteile sowie Einschränkungen. Diese sind auf Grund der Funktionalitäten der Wearables gegeben und resultieren aus Eingabe- und Ausgabemöglichkeiten der Geräte. Zudem haben sowohl der Einsatz in industriellen Umgebungen als auch die menschlichen Bedürfnisse und Fähigkeiten Einfluss auf die mögliche Zuordnung von Tätigkeit und Wearable. Innerhalb des Projektes wird die Vielzahl von Möglichkeiten in eine Methodik überführt, um damit einen sinnvollen Einsatz des Gesamtsystems unter Einbezug des Menschen zu gewährleisten.

In drei Szenarien aus unterschiedlichen Bereichen wird das SmARPro-Systemkonzept umgesetzt und die angestrebte Unterstützung der Arbeitsprozesse des Menschen sowie die Verbesserung der Einbindung des Menschen in die Unternehmensprozesse betrachtet. Die Umsetzung erfolgt in den Use-Cases Produktion, Logistik und Werkzeugmanagement. Stellvertretend für die anderen Bereiche wird nachfolgend der Einsatz des SmARProSystems in der Logistik näher betrachtet. Hier werden sowohl die Shopfloor-Ebene als auch die operative Schicht jeweils in Verbindung mit unterschiedlichen Rollen. Dadurch kann eine Allgemeingültigkeit für die Einsatzmöglichkeiten des SmARPro-Systems auch in anderen Branchen und Bereichen abgeleitet werden. 


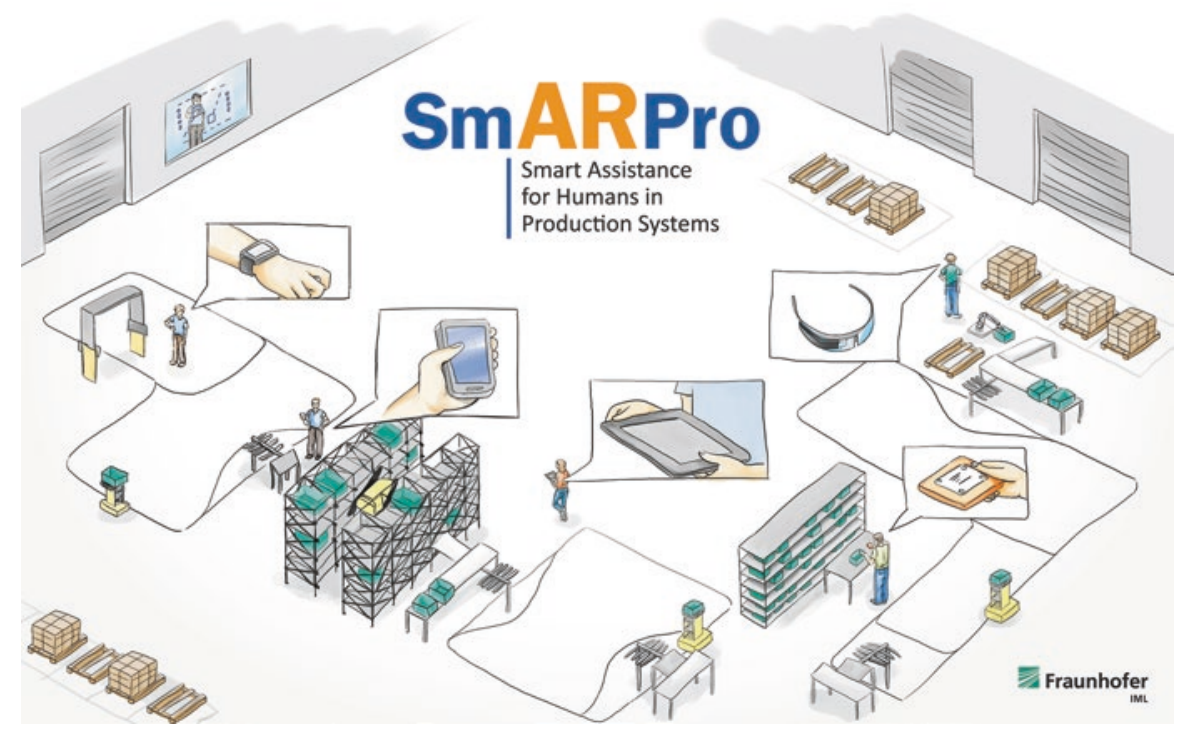

Abb. 5.1 Anwendungsfall Intralogistik (Quelle: Fraunhofer IML)

Der Gesamtprozess in der Logistik, wie in Abb. $5.1 \mathrm{zu}$ sehen ist, setzt sich im Wesentlichen aus Wareneingang, Lagerung, Kommissionierung, Verpackung, Bereitstellung und dem Transport, z. B. mit fahrerlosen Transportfahrzeugen als verbindendes Element zwischen den verschiedenen Bereichen, zusammen. Die anfallenden Informationen entlang der Prozesskette werden bedarfsgerecht aufbereitet. An den entsprechenden Orten werden sie dann den jeweiligen Personen mit bestimmten Rollen mittels Augmented Reality direkt im Sichtfeld angezeigt. Die Informationen werden nicht nur perspektivisch über der Maschine oder dem Objekt von Interesse präsentiert, es erfolgt auch eine Einbeziehung rollenbezogener Inhalte. Wird beispielsweise der Kommissionierprozess betrachtet, so können dem Kommissionierer, der sich vor einem Regal befindet und einen Kommissionierauftrag bearbeitet, Instruktionen über das Wearable geliefert werden. Im Sichtfeld des Benutzers wird die Greifrichtung mithilfe von Pfeilen angezeigt, die mit dem Regal passend überlagert werden. Blickt der Kommissionierer schließlich auf das Zielfach, werden ihm weitere Informationen wie der zu entnehmende Artikel und die Stückzahl eingeblendet.

Ein weiteres Beispiel ist in Abb. 5.2 zu sehen. So werden nicht nur Prozessinformationen an die Mitarbeiter weitergeleitet, es können auch Statusinformationen für ein Monitoring der einzelnen Geräte angezeigt werden. Nähert sich beispielsweise der Wareneingangskontrolleur dem RFID-Gate, so erhält er auf seiner Datenbrille direkt die für seine Rolle notwendigen Informationen des Gates. Schnell und auf einen Blick werden ihm der aktuelle Lieferant sowie Liefermenge und Artikelnummern dargestellt.

Auch können durch SmARPro Fehlerreaktionszeiten verbessert werden. Tritt beispielsweise ein Fehler an einer Maschine auf, so werden die Informationen an den 
Abb. 5.2 Blick durch eine Datenbrille am Wareneingang (Quelle: Fraunhofer IML)

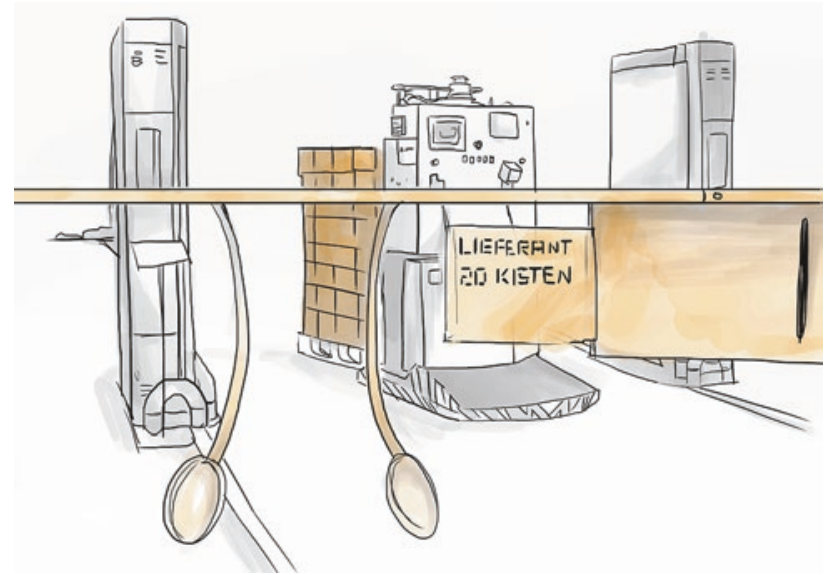

entsprechenden Mitarbeiter, bspw. den Instandhalter, weitergeleitet. Neben der Fehlermeldung und Informationen zur Fehlerbehebung wird dem Mitarbeiter auch der Weg zum Gewerk in Abhängigkeit seines eigenen Ortes angezeigt.

\subsection{Betroffene Tätigkeiten}

Typische Tätigkeiten in der Intralogistik finden sich in der Kommissionierung, am Wareneingang, am Warenausgang, in der Instandhaltung, in der Verpackung sowie in der Qualitätssicherung, der Lagerverwaltung und der Betriebsleitung wieder. Innerhalb des folgenden Abschnittes werden die jeweiligen Tätigkeitsfelder beschrieben und hinsichtlich möglicher Veränderungen durch den Einsatz von SmARPro betrachtet.

Die Arbeit der Zukunft wird stark von Mensch-Technik-Schnittstellen geprägt sein. Wearables können im logistischen Umfeld auf verschiedene Arten eingesetzt werden. Diese Geräte werden in der Hand (Smartphone/Tablet) gehalten, oder auf dem Kopf (Datenbrille) bzw. am Arm (Smartwatch) getragen. Die Ausprägung der unterschiedlichen Wearables bedingt den Einsatz für die jeweiligen Tätigkeiten. Primär werden diese technischen Geräte eingesetzt, um dem Menschen Informationen bedarfsgerecht zu vermitteln und den Arbeitsablauf zu unterstützen.

Am Wareneingang und am Warenausgang bestehen die Aufgaben aus Tätigkeiten mit organisatorischem und physischem Anteil. Hier müssen Lieferscheine in der Anlieferung begutachtet und mit der erhaltenen Ware abgeglichen werden. Bei Fehllieferungen werden Meldungen an Verwaltungssysteme weitergeleitet und Lieferungen ggf. abgelehnt. Speziell am Warenausgang muss die Ladungssicherung garantiert werden. Physische Arbeiten umfassen die Bewegung von Paletten mit Staplern von und zu Zwischenlagerorten.

Zukünftig wird der Mitarbeiter am Wareneingang und -ausgang in seiner Tätigkeit durch das im Projekt SmARPro entwickelte System unterstützt. So wird beispielsweise der eingescannte oder durch ein RFID-Gate elektronisch erfasste Lieferschein mit den 
Bestelldaten im Warenwirtschaftssystem abgeglichen. Daraufhin erhält der Mitarbeiter aggregierte Informationen zu den gelieferten und den bestellten Waren. Erkennt das SmARPro-System Unregelmäßigkeiten, wird der Mitarbeiter darüber informiert und mit Handlungsempfehlungen hinsichtlich einer möglichen Reklamation und Rücksendung der Waren unterstützt. Entdeckt der Mitarbeiter bei der visuellen Prüfung der Ware oder beim Auswerten digitaler Daten von in Ladungsträgern integrierten Sensoren Auffälligkeiten, so kann er diese über sein Wearable im System vermerken und ggf. direkt eine detaillierte Überprüfung, eine Reklamation oder einen internen Austausch der Ware anstoßen.

Die Lagerverwaltung beinhaltet alle Aspekte, die mit dem Lager zusammenhängen. Dies umfasst auf der einen Seite die Bestandsverwaltung und einen Überblick über nachzubestellende Waren. Auf der anderen Seite umfasst dies die Durchführung von Ein- und Auslagerungen unter der Berücksichtigung möglicher Lagerzonen, die das Lager nach schnell und langsam drehenden Artikeln aufteilt.

Auch bei dieser Tätigkeit kann der Mitarbeiter durch das SmARPro-System besser in die bestehenden Prozesse integriert werden. Über sein Wearable erhält er auf einen Blick alle relevanten Informationen über das Lager. Mögliche Fehler und Lösungsvorschläge werden ihm darauf angezeigt. Einerseits kann er hierüber Ein- und Auslagerungsaufträge anstoßen, andererseits ermöglicht der direkte Zugriff über Wearables schnelle und unkomplizierte Eingriffe in mögliche Lagerstrategien und damit verbundene Einsparungen hinsichtlich Zugriffszeiten.

In der Kommissionierung sind verstärkt körperliche Tätigkeiten anzutreffen. Hier werden einzelne Artikel aus unterschiedlichen Orten im Warenlager in Verpackungseinheiten oder Behälter zusammengestellt. Hierfür muss sich der Kommissionierer im Lager fortbewegen, also zu verschiedenen Orten laufen oder fahren. Eine wichtige Tätigkeit ist hier die Navigation in Bereichen und Regalgassen und zusätzlich die Orientierung vor Regalen, um zu dem korrekten Artikel zu finden.

Durch die Verwendung von Wearables und durch die SmARPro-Plattform kann der Prozess der Kommissionierung für den Mitarbeiter stark vereinfacht werden. Über sein Wearable erhält er Informationen zum aktuellen Kommissionierauftrag und wird schrittweise durch den Prozess geführt. Sowohl die Darstellung der zu kommissionierenden Artikel und deren Anzahl sowie die Richtung zum Lagerplatz des Artikels in Abhängigkeit von seiner aktuellen Position werden ihm auf dem Wearable angezeigt. Durch das Scannen oder die optische Identifikation der kommissionierten Artikel kann ein Abgleich mit dem im Kommissionierauftrag geforderten Artikel erfolgen. Dies ermöglicht die frühzeitige Erkennung von Fehlern und eine umgehende Benachrichtigung des Kommissionierers noch während des Prozesses. Weiterhin kann der Kommissionierer den Nachschub von Waren und Leerbehältern aus dem Lager anstoßen. Hier gleicht das SmARPro-System zunächst mit dem Lagerverwaltungssystem ab, ob die Ware im Lager vorhanden ist. Falls ja, so wird ein Auslagerungsprozess der Ware angestoßen. Ist dies nicht der Fall, erhält der Kommissionierer eine Warnmeldung, dass seine angeforderte Ware nicht vorrätig ist, und er erhält die Möglichkeit, einen Bestellauftrag direkt über sein Wearable auszulösen. Gerade in diesem Szenario bieten sich Datenbrillen besonders an, da der Mitarbeiter die Hände dauerhaft frei hat und sich so auf die Kommissioniertätigkeit konzentrieren kann. 
In der Verpackung muss der Mensch ebenfalls körperliche Arbeit durchführen. Aber auch die Verwendung von passenden Verpackungseinheiten und die optimale Anordnung der Verpackungseinheiten auf Paletten stellen den Verpacker vor besondere Herausforderungen. Hier muss entschieden werden, welches Packmittel verwendet werden soll und wie Packstücke unterschiedlicher Größe und unterschiedlichen Gewichts optimal angeordnet werden müssen. Teils gibt es dafür Packvorschriften, teils werden die Packstücke nach Erfahrung arrangiert.

Durch SmARPro sollen diese Herausforderungen minimiert werden. Über sein Wearable erhält der Mitarbeiter Bestellinformationen, welche neben den zu verpackenden Artikeln auch Informationen zur Auswahl des Packmittels sowie über das zugehörige Packschema beinhalten. Schritt für Schritt wird der Mitarbeiter durch den Verpackungsprozess geleitet und über Augmented Reality die Einhaltung des Packschemas gewährleistet. Wie auch in der Kommissionierung ist in der Verpackung die Verwendung von Datenbrillen besonders sinnvoll.

Die Instandhaltung befasst sich mit der Analyse, Reparatur, Optimierung und Aufrechterhaltung von technischen Anlagen. Instandhalter arbeiten mit Werkzeugen, Ersatzteilen und Konstruktionsplänen, um Technologien im produktiven Zustand zu halten und somit die Verfügbarkeit der Anlagen zu gewährleisten, Störungen zu beseitigen und die Betriebssicherheit zu garantieren. Fehlerhafte Maschinen müssen schnellstmöglich repariert werden und aus betriebswirtschaftlicher Sicht kurzfristig wieder einsatzbereit sein. Die Aufgaben des Instandhalters erstrecken sich von einfachen Batteriewechseln über kleinere Reparaturen bis hin zu komplexen Komponentenwechseln bei Maschinen.

Der Mitarbeiter soll in Zukunft durch SmARPro auch bei dieser Tätigkeit besser unterstützt werden. Alle relevanten Informationen werden dem Instandhalter auf seinem Wearable dargestellt. Er wird über Fehler an Maschinen rechtzeitig informiert, sodass er schnellstmöglich reagieren kann. Bei der Wartung und Fehlerbehebung können ihm technische Dokumente, unterstützende Modelldaten, aber auch Schritt für Schritt Anleitungen angezeigt werden. Durch den Einsatz von Augmented Reality können dem Mitarbeiter umfangreiche Unterstützungsinformationen direkt an der $\mathrm{zu}$ wartendenden Maschine visualisiert werden. Dies verringert im Bereich der Instandhaltung die Einarbeitungszeit und ermöglicht eine Assistenz speziell im Kontext unregelmäßig auftretender Arbeiten.

Der Betriebsleiter (in der Intralogistik häufig auch Lagerleiter) benötigt den Gesamtüberblick über alle intralogistischen Prozesse sowie über mögliche Probleme innerhalb eines bestimmten Tätigkeitsfeldes. Er muss Zusammenhänge erkennen und schnell auf Anfragen bzw. Problemstellungen reagieren können. Zudem kommuniziert er mit den anderen Mitarbeitern im Lager und übernimmt Teile der Arbeitsorganisation.

Auch für den Betriebsleiter liefert SmARPro Ansätze zur Unterstützung. Die notwendigen Informationen zu aktuellen Prozessen werden ihm verständlich und in den entsprechenden Zusammenhängen auf seinem Wearable angezeigt. Dies umfasst beispielsweise Informationen zu aktuellen Prozesszeiten oder offenen Entscheidungen, die seiner Zustimmung bedürfen. Darüber hinaus kann der Betriebsleiter umgehend über mögliche Probleme in den jeweiligen Betriebsbereichen informiert werden und daraufhin direkt über sein Wearable mit den betreffenden Mitarbeitern in Kontakt treten. Bei Werksbesichtigungen kann der Betriebsleiter an Ort und Stelle kontextbezogene Kennzahlen zu Prozessen und Leistungsdaten zu Maschinen und Anlagen abfragen. Besonders adäquate 
Darstellungsmodelle gewährleisten, dass er alle relevanten Informationen erhält, ohne durch die Menge der angezeigten Informationen überfordert zu werden.

Die Qualitätssicherung zieht sich durch die einzelnen Tätigkeiten und manifestiert sich in der Begutachtung von Dokumenten, Waren, Ladungsträgern und Verpackungsmaterialien sowie der Korrektheit der Ablageorte und Artikelbestände. Durch die Digitalisierung der Prozessdaten und die Verknüpfung von Informationen im SmARPro-System wird die durchgängige Unterstützung in der Qualitätssicherung prozessübergreifend gewährleistet.

\subsection{Auswirkungen auf die betrieblichen Funktionen}

Die kontextbezogene Informationsdarstellung auf den Wearables hat einen großen Einfluss auf die Tätigkeiten des betrachteten Szenarios. Während das System keine nennenswerten Auswirkungen im Personalbedarf der einzelnen betrieblichen Funktionen hat, zeigen sich Auswirkungen auf die Tätigkeitsausführung. In der nachfolgenden Tabelle sind die Auswirkungskriterien in Bezug auf die einzelnen Tätigkeiten aufgeführt.

\begin{tabular}{l|l|l|l|l|l|l|l}
\hline & $\begin{array}{l}\text { Kom- } \\
\text { missio- } \\
\text { nierung }\end{array}$ & $\begin{array}{l}\text { Lager- } \\
\text { verwal- } \\
\text { tung }\end{array}$ & $\begin{array}{l}\text { Verpa- } \\
\text { ckung }\end{array}$ & $\begin{array}{l}\text { Waren- } \\
\text { eingangs- } \\
\text { kontrolle }\end{array}$ & $\begin{array}{l}\text { Quali- } \\
\text { tätssi- } \\
\text { cherung }\end{array}$ & $\begin{array}{l}\text { Instand- } \\
\text { haltung }\end{array}$ & $\begin{array}{l}\text { Betriebs- } \\
\text { leitung }\end{array}$ \\
\hline Bedarf & 0 & 0 & 0 & 0 & 0 & 0 & 0 \\
\hline Problemlösung & -- & -- & -- & -- & -- & -- & - \\
\hline $\begin{array}{l}\text { Monotone } \\
\text { Aufgaben }\end{array}$ & 0 & 0 & 0 & 0 & 0 & - & - \\
\hline $\begin{array}{l}\text { Komplexe } \\
\text { Aufgaben }\end{array}$ & 0 & 0 & 0 & 0 & - & + & 0 \\
\hline Planen & - & - & - & - & - & - & - \\
\hline Kontrolle & + & + & + & + & + & ++ & ++ \\
\hline $\begin{array}{l}\text { Lernen, } \\
\text { informell }\end{array}$ & + & + & + & + & + & + & + \\
\hline Lernen, formell & - & - & - & - & - & - & + \\
\hline $\begin{array}{l}\text { Selbstbestim- } \\
\text { mung }\end{array}$ & - & - & - & 0 & + & + & 0 \\
\hline Optimierung & + & + & + & + & + & + & + \\
\hline Kooperation & ++ & ++ & ++ & ++ & ++ & ++ & ++ \\
\hline $\begin{array}{l}\text { Kommunika- } \\
\text { tion }\end{array}$ & + & + & + & + & + & + & + \\
\hline $\begin{array}{l}\text { Interdiszipli- } \\
\text { narität }\end{array}$ & + & + & + & + & + & + & + \\
\hline IT-Kenntnisse & 0 & 0 & 0 & 0 & 0 & 0 & 0 \\
\hline Legende: & + & + & + & + & + & + & + \\
\hline
\end{tabular}

Legende: -- wird viel weniger, - wird weniger, 0 bleibt gleich, + wird mehr, ++ wird viel mehr. 
Den Mitarbeitern werden durch das SmARPro-System prozessspezifische Lösungsvorschläge unterbreitet. Hierdurch sinkt der Bedarf einer eigenständigen Lösungsfindung des einzelnen Mitarbeiters. Im Kontext einfacher Tätigkeiten ergeben sich keine nennenswerten Änderungen hinsichtlich der Verteilung monotoner und komplexer Aufgaben, da sich der generelle Prozessablauf dieser Tätigkeiten nicht verändert. Hingegen sinkt bei variablen Tätigkeiten wie der Instandhaltung oder der Betriebsleitung der Anteil monotoner Arbeiten. Dies lässt sich einerseits auf einen umfangreicheren Tätigkeitsbereich zurückführen. Andererseits ergeben sich durch den Einsatz von Wearables verbesserte Kontroll- und Steuerfunktionalitäten. Generell sinkt aufgrund der Informationsbereitstellung des SmARPro-Systems der Bedarf an Planungsaktivitäten der Mitarbeiter. Hierdurch erhält der Mitarbeiter eine erhöhte Entscheidungs- und Kontrollkompetenz. Außerdem ist eine Verschiebung vom formellen zum informellen Lernen zu verzeichnen. Durch Hilfestellungen und Anweisungen über die Wearables ermöglicht SmARPro ein ,learning by doing“. Die Reduktion der Fehler im Prozess führt bei einigen einfachen Tätigkeiten (lineare Abarbeitung) zu einer Verringerung der Selbstbestimmung. Bei komplexeren Tätigkeiten kann jedoch eine Zunahme derselben erfolgen. Prozesse und Tätigkeiten werden durch die Assistenz der Wearables optimiert. Zudem werden Fehler minimiert. Weiterhin lässt sich eine Verbesserung der Kooperation in Teams sowie der Kommunikation zwischen den Mitarbeitern durch den Einsatz der Wearables und der damit verbundenen direkten Interaktion der Mitarbeiter über Hierarchiegrenzen hinweg erkennen. Durch die Möglichkeit des schnellen Rollenwechsels sowie der Ausführung tätigkeitsübergreifender Prozesse (z. B. Auslagerungsbefehl durch den Kommissionierer) steigt in allen Bereichen die Interdisziplinarität. Die Verwendung einfacher und selbsterklärender Benutzeroberflächen ermöglicht den Einsatz des SmARPro-Systems ohne gesteigerte ITKenntnisse der Anwender.

Nach Vorstellung der einzelnen Tätigkeiten und der Bewertung ihrer Veränderungen anhand verschiedener Kriterien wird im Folgenden auf die allgemeinen Auswirkungen des Einsatzes des SmARPro Gesamtsystems eingegangen.

Auch wenn sich die Prozesse an sich nicht gravierend verändern, kommt es zu einer Prozessbeschleunigung. Teilschritte wie beispielsweise das Abrufen von Auftragsdaten bei der Kommissionierung an externen Systemen oder auf Papier entfallen, da das SmARProSystem weiß, wo und in welchem Arbeitsschritt sich der jeweilige Mitarbeiter befindet.

Die Prozessbeschleunigung geht einher mit einer schnelleren Fehlerbehebung und Fehlerreduktion. Durch ein direktes Feedback am jeweiligen Wearable können Fehler leichter und schneller erkannt werden. Wird eine Packfolge nicht eingehalten, weist das Wearable den Mitarbeiter darauf hin. Nicht nur das Beheben von Fehlern wird durch SmARPro erleichtert, sondern auch ihre Reduktion. Der Mitarbeiter wird abhängig von seinen bisherigen Erfahrungen und seinen persönlichen Stärken und Schwächen durch die verschiedenen Prozesse geleitet. Zudem kann durch den Einsatz von AR-fähigen Wearables die vom Menschen wahrgenommene Komplexität der Prozesse minimiert werden. Die 
weiterhin komplexen Abläufe werden in einzelne Arbeitsschritte aufgeteilt und für jeden dieser Arbeitsschritte erhält der Mitarbeiter Hilfestellungen sowie ein Feedback bei der Ausführung. Dies führt zu einer Verringerung der kognitiven Belastung, wodurch sich eine Arbeitserleichterung für den Mitarbeiter ergibt. Hierdurch kann die Qualifikation der Mitarbeiter für einzelne Tätigkeiten geringer ausfallen. Jedoch ermöglicht das SmARProGesamtsystem auch die Verschmelzung von Tätigkeitsfeldern. So kann ein und derselbe Mitarbeiter in verschiedenen Kontexten und Rollen arbeiten. Hierdurch ergeben sich für den Mitarbeiter eine erhöhte Flexibilität hinsichtlich seines Einsatzes sowie eine abwechslungsreiche Tätigkeit.

Neben der Verringerung der kognitiven Belastung durch SmARPro wird auch die körperliche Belastung minimiert. Die einzelnen Arbeitsschritte sind nicht nur prozessoptimierend ausgelegt, sondern auch ergonomisch, sodass Fehlhaltungen oder beispielsweise ein Verheben beim Palettieren minimiert werden. Neben Kosteneinsparungen durch Fehlerreduktion werden Kosten auch beim Einsatz von Ressourcen verringert. Oft verfügen Unternehmen über eine breite Produktpalette und über eine begrenzte Menge an Versandmaterial. Dabei werden von den Mitarbeitern häufig zu große Verpackungen ausgewählt und zusätzliches Füllmaterial verwendet, um Beschädigungen an den Artikeln zu vermeiden. SmARPro soll hier Abhilfe schaffen, indem dem Mitarbeiter auf seinem Wearable, abhängig von dem zu versendenden Artikel, die passende Verpackung sowie die Menge an zu verwendendem Füllmaterial angezeigt wird.

\subsection{Zusammenfassende Betrachtung der Auswirkungen}

\begin{tabular}{l|l}
\hline Beschreibungskriterium & Erläuterung \\
\hline Arbeitsaufgabe & $\begin{array}{l}\text { Die Tätigkeiten der Akteure werden durch eine Reduktion der } \\
\text { Informationssuche erleichtert. Die bedarfsgerechte Anzeige der } \\
\text { Informationen erfolgt im Sichtbereich des Mitarbeiters }\end{array}$ \\
\hline Arbeitstätigkeiten & $\begin{array}{l}\text { Die kognitive Belastung wird durch Hilfestellungen sowie fein- } \\
\text { granulare Prozessschritte reduziert. Der Fokus der Tätigkeiten ver- } \\
\text { schiebt sich vom Planen hin zum Kontrollieren und Entscheiden }\end{array}$ \\
\hline Arbeitsplatz/Arbeitsraum & $\begin{array}{l}\text { Das SmARPro-System ermöglicht einen Tätigkeitswechsel } \\
\text { sowie einen Wechsel des Arbeitsplatzes. Hierdurch ergibt sich } \\
\text { eine erhöhte Flexibilität der Arbeitsgestaltung des jeweiligen } \\
\text { Mitarbeiters }\end{array}$ \\
\hline Arbeitsmittel & $\begin{array}{l}\text { Als zentrales Element kommen mehr Mensch-Maschine- } \\
\text { Schnittstellen in Form von elektronischen Endgeräten (Weara- } \\
\text { bles) zum Einsatz }\end{array}$ \\
\hline Arbeitsorganisation & $\begin{array}{l}\text { Die Arbeitsorganisation ist dynamischer. Die Skalierbarkeit } \\
\text { der menschlichen Ressourcen nimmt durch den vereinfachten } \\
\text { Arbeitsplatzwechsel zu. Die Koordinierung von Arbeitsgruppen } \\
\text { wird vereinfacht }\end{array}$ \\
\hline
\end{tabular}




\begin{tabular}{l|l}
\hline Beschreibungskriterium & Erläuterung \\
\hline Arbeitsumgebung & $\begin{array}{l}\text { Die Einsatzbereiche der Logistikarbeiter werden erweitert } \\
\text { und die Verfügbarkeit der Informationen wird verbessert }\end{array}$ \\
\hline Qualifikation/Kompetenz & $\begin{array}{l}\text { Im Kontext einfacher Tätigkeiten sinkt der Bedarf an zusätz- } \\
\text { lichen Qualifizierungsmaßnahmen. Durch zusätzliche Informa- } \\
\text { tionsanzeige erfolgt ein Kompetenzgewinn in allen Tätigkeiten }\end{array}$ \\
\hline Führung & $\begin{array}{l}\text { Es resultiert eine verbesserte Kommunikation zwischen den } \\
\text { Hierarchieebenen. Dadurch wird die unmittelbare Ausführung } \\
\text { von Arbeitsaufträgen ermöglicht }\end{array}$ \\
\hline
\end{tabular}

Open Access Dieses Kapitel wird unter der Creative Commons Namensnennung 4.0 International Lizenz (http://creativecommons.org/licenses/by/4.0/deed.de) veröffentlicht, welche die Nutzung, Vervielfältigung, Bearbeitung, Verbreitung und Wiedergabe in jeglichem Medium und Format erlaubt, sofern Sie den/die ursprünglichen Autor(en) und die Quelle ordnungsge-mäß nennen, einen Link zur Creative Commons Lizenz beifügen und angeben, ob Änderungen vorgenommen wurden.

Die in diesem Kapitel enthaltenen Bilder und sonstiges Drittmaterial unterliegen ebenfalls der genannten Creative Commons Lizenz, sofern sich aus der Abbildungslegende nichts anderes ergibt. Sofern das betreffende Material nicht unter der genannten Creative Commons Lizenz steht und die betreffende Handlung nicht nach gesetzlichen Vorschriften erlaubt ist, ist für die oben aufgeführten Weiterverwendungen des Materials die Einwilligung des jeweiligen Recht-einhabers einzuholen.

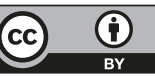

